University of Nebraska - Lincoln

DigitalCommons@University of Nebraska - Lincoln

2011

Spatial Patch Occupancy Patterns of the Lower Keys Marsh Rabbit

\author{
Mitchell J. Eaton \\ Phillip T. Hughes \\ James D. Nichols \\ Anne Morkill \\ Chad Anderson
}

Follow this and additional works at: https://digitalcommons.unl.edu/usgsstaffpub

Part of the Geology Commons, Oceanography and Atmospheric Sciences and Meteorology Commons, Other Earth Sciences Commons, and the Other Environmental Sciences Commons

This Article is brought to you for free and open access by the US Geological Survey at DigitalCommons@University of Nebraska - Lincoln. It has been accepted for inclusion in USGS Staff -- Published Research by an authorized administrator of DigitalCommons@University of Nebraska - Lincoln. 


\title{
Spatial Patch Occupancy Patterns of the Lower Keys Marsh Rabbit
}

\author{
MITCHELL J. EATON, ${ }^{\mathbf{1}, 2}$ Patuxent Wildlife Research Center, United States Geological Survey, 12100 Beech Forest Road, Laurel, MD 20708, USA \\ PHILlIP T. HUGHES, National Key Deer Refuge, United States Fish and Wildlife Service, 28950 Watson Boulevard, Big Pine Key, FL 33043, \\ $U S A$ \\ JAMES D. NICHOLS, Patuxent Wildlife Research Center, United States Geological Survey, 12100 Beech Forest Road, Laurel, MD 20708, USA \\ ANNE MORKILL, National Key Deer Refuge, United States Fish and Wildife Service, 28950 Watson Boulevard, Big Pine Key, FL 33043, USA \\ CHAD ANDERSON, National Key Deer Refuge, United States Fish and Wildlife Service, 28950 Watson Boulevard, Big Pine Key, FL 33043, USA
}

\begin{abstract}
Reliable estimates of presence or absence of a species can provide substantial information on management questions related to distribution and habitat use but should incorporate the probability of detection to reduce bias. We surveyed for the endangered Lower Keys marsh rabbit (Sylvilagus palustris hefneri) in habitat patches on 5 Florida Key islands, USA, to estimate occupancy and detection probabilities. We derived detection probabilities using spatial replication of plots and evaluated hypotheses that patch location (coastal or interior) and patch size influence occupancy and detection. Results demonstrate that detection probability, given rabbits were present, was $<0.5$ and suggest that naïve estimates (i.e., estimates without consideration of imperfect detection) of patch occupancy are negatively biased. We found that patch size and location influenced probability of occupancy but not detection. Our findings will be used by Refuge managers to evaluate population trends of Lower Keys marsh rabbits from historical data and to guide management decisions for species recovery. The sampling and analytical methods we used may be useful for researchers and managers of other endangered lagomorphs and cryptic or fossorial animals occupying diverse habitats. (c) 2011 The Wildlife Society. ${ }^{\dagger}$
\end{abstract}

KEY WORDS detection probability, Florida Keys, marsh rabbit, monitoring, occupancy, Sylvilagus palustris hefneri.

Managing natural resources often requires the use of models to understand the impact of management on resource dynamics and predict the consequences of alternative actions to make the best decisions relative to management objectives (Williams et al. 2002, Pielke and Conant 2003). The abundance of a target organism is commonly used as a state variable for predictive models in evaluating the outcome of management actions (MacKenzie and Nichols 2004, Conroy et al. 2008). The proportion of sites occupied by a focal species, however, is often closely correlated with abundance and may be preferable as a state variable for conservation management programs (Hanski 1991, Gotelli 2000, Carrete et al. 2002, MacKenzie and Kendall 2002, Royle et al. 2005). Estimation of occupancy can be especially appropriate when the focus is on rare or threatened species or on organisms occurring at the limits of their natural distribution and for which estimating the extent of occupied range is of critical importance to management (Mace and

Received: 8 August 2010; Accepted: 16 November 2010; Published: 4 May 2011

\footnotetext{
${ }^{\dagger}$ This article is a US Government work and, as such, is in the public domain in the United States of America.

${ }^{1}$ E-mail: meaton@usgs.gov

${ }^{2}$ Present Address: USGS-Patuxent Wildlife Research Center, 12100 Beech Forest Rd., Laurel, MD 20708, USA.
}

Lande 1991, Doherty et al. 2003). Occupancy is a central state variable in meta-population studies, where the principal concern is the extinction and colonization of local sites (patches) rather than estimates of abundance or density (Hanski 1991, Komonen et al. 2008). Specifically, singleand multiple-species incidence functions and patch occupancy models were developed to understand the influence of ecological factors on probabilities of occurrence, extinction, and colonization (e.g., Diamond 1975, Hanski 1992, Ovaskainen 2002). In such applications, estimates of occupancy rates and dynamic parameters may be biased if the probability of detecting the species is $<1.0$, that is, if the focal species was sometimes present but was not detected (MacKenzie and Kendall 2002, MacKenzie et al. 2004). Management programs that use occupancy as a state variable for decision making, but do not account for detection probability in monitoring design and analysis, will often produce sub-optimal decisions and result in slower learning about system behavior. Considerations when using occupancy as a surrogate for abundance should include possible trade-offs between reduced cost and effort and the strength of the correlation between abundance and area occupied (MacKenzie and Kendall 2002, MacKenzie et al. 2003, MacKenzie and Nichols 2004, Kendall and White 2009, but see Royle et al. 2005). As highlighted above, there are many situations in which occupancy is preferred over abundance as the state variable of choice. 
Recent applications of occupancy models have focused attention on species occupancy in the context of management by explicitly considering biases generated by imperfect detection and the effects of management actions on demographic rates of colonization and extinction (e.g., Kroll et al. 2008; Martin et al. 2009, 2010). This approach allows conservation efforts to be more targeted, focusing on those factors and vital rates having the greatest impact relative to management goals.

The Lower Keys marsh rabbit (Sylvilagus palustris hefneri; marsh rabbit, hereafter) is a sub-species endemic to the lower Florida Keys. The species was historically widespread and abundant across its range but is now limited to only a subset of islands due to habitat fragmentation and degradation, predation by domestic cats, and road mortality (U.S. Fish and Wildlife Service 1990, 2007). These factors resulted in the marsh rabbit being listed as federally endangered in 1990 , but continued succession from marsh to sub-optimal habitat, predation by native and non-native predators, and impacts from sea-level rise and hurricanes have perpetuated the decline of this species. Current suitable habitat is considered to be restricted to small, isolated patches with limited connectivity (Schmidt 2009).

The proportion of sites occupied $(\psi)$ by marsh rabbits appears to be an appropriate state variable for use in prescribing management actions (i.e., state-dependent decisions) and for monitoring. Methods for assessing the status, abundance, or detection probabilities of marsh rabbit populations, such as mark-recapture or radio telemetry, were considered to be cost prohibitive and logistically infeasible given the current spatial distribution and goal of monitoring a large number of populations, including those affected by management. The use of occupancy as a state variable is also consistent with recovery objectives and management guidelines for the subspecies. These guidelines designate the basic unit of management as the habitat patch, define extinctions as "the loss of [Lower Keys marsh rabbit] in a patch" (i.e., present or absent), and recommend monitoring via fecal pellet detection to provide confirmation of rabbit presence (U.S. Fish and Wildlife Service 2007).

Marsh rabbits have been surveyed repeatedly in our study area (e.g., Howe 1988, Forys and Humphrey 1997, Faulhaber et al. 2007), but previous studies were not designed to account for imperfect detection when making inference about occupancy and distribution. Because monitoring will unlikely detect marsh rabbits perfectly (i.e., with probability 1.0), occupancy estimates will be biased unless detection probability is considered explicitly. To improve inference on the relationship between habitat and occupancy, we implemented a new survey method designed to estimate detection probability of marsh rabbits. Although our intent was to inform decisions on habitat management, we did not explicitly consider the outcomes of specific management alternatives in this initial work but instead examined the influence of habitat characteristics on occupancy rates with the belief that this represents a first step in guiding management decisions and subsequent monitoring activities. Actions proposed to increase marsh rabbit occupancy are generally focused on habitat manipulation (fire and mechanical treatment) and reduction of non-native predators. The ability of fire to modify habitat and affect marsh rabbit vulnerability to predators is strongly influenced by vegetation characteristics. As such, we hypothesized that vegetation class directly influences rabbit occupancy and the ability of management to effect change in habitat and therefore, indirectly, elicit a response in occupancy. Quantifying the relationship between marsh rabbit occupancy rates and habitat characteristics will allow managers to direct habitat restoration actions to the most appropriate locations for maximizing the distribution of rabbit metapopulations and, therefore, increasing population persistence. Monitoring changes in occupancy following management actions will also provide the means to reduce uncertainty in the parameters governing colonization and extinction dynamics via model updating as prescribed by formal adaptive management (Williams et al. 2002).

Our objectives were to: 1) evaluate the efficacy of pellet survey methods and occupancy models for inference on marsh rabbit occupancy and 2) test a priori hypotheses about the influence of patch size and habitat characteristics on site occupancy and detection probabilities. We defined patch habitat characteristics using patch location (inland vs. coastal) as a proxy measure for the combined influence of vegetation type and exposure to seawater influx. Based on the results of previous surveys (Faulhaber et al. 2007, U.S. Fish and Wildlife Service 2007, Schmidt 2009), we anticipated that coastal sites would have a lower incidence of patch occupancy (i.e., smaller $\psi$ ) than would inland sites, even though the former studies did not account for issues of imperfect detection. We also predicted that rabbit detectability would vary with site location due to differences in vegetation between coastal and inland habitats, with a lower probability of detection in coastal settings due to denser ground cover. Habitat area or edge effects have been shown to affect the risk of patch extinction and colonization in numerous species (Hanski 1991, Taylor 1991, Komonen et al. 2008, Collins et al. 2009). We predicted probability of occupancy to increase with total patch size.

\section{STUDY AREA}

The study area included 5 islands in the Florida Keys, USA, a chain of islands extending southwest from the southern tip of the Florida mainland peninsula to the Dry Tortugas (Fig. 1). Marsh rabbit survey plots were located in the lower archipelago: No Name Key, Big Pine Key, Upper Sugarloaf Key, Lower Sugarloaf Key, and the Saddlebunch Keys. Islands included in the study range from 327 ha (Howe Key) to 2,357 ha (Big Pine Key). Although the Florida Keys were sub-tropical, the climate was considered tropical with most rainfall occurring from June to October and drier months from November to May (Ross et al. 1992). Major vegetation types in the Lower Keys transitioned along an elevational gradient, from tidal wetlands adjacent to coastlines to forest and freshwater wetlands in the upland interior. Low-lying tidal wetlands areas were dominated by swamps containing red (Rhizophora mangle), white (Laguncularia racemosa), and black mangrove (Avicennia germinans). A wetland transition 


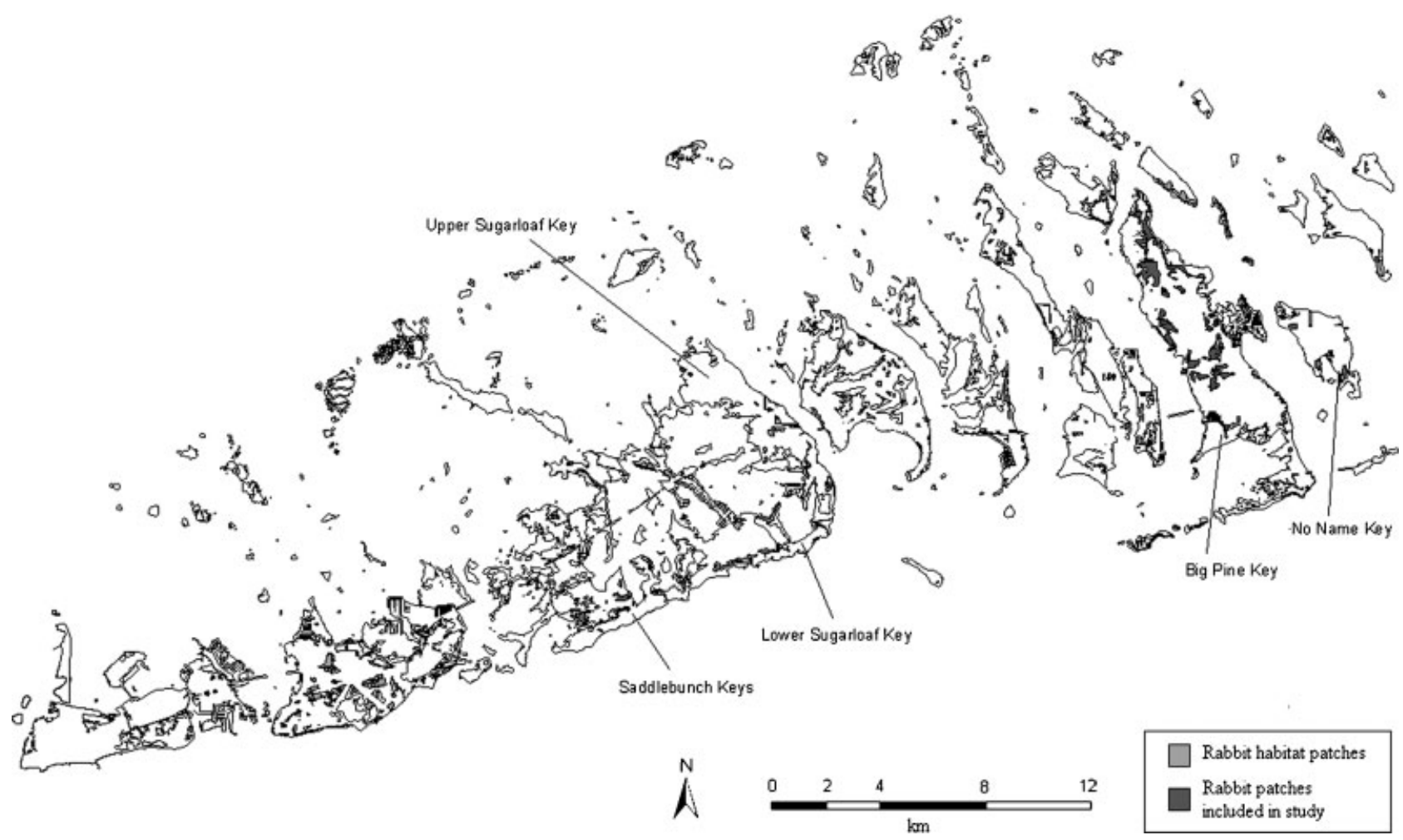

Figure 1. Map of the lower Florida Keys, USA. Known habitat patches of Lower Keys marsh rabbit are indicated in light gray; 36 habitat patches that were included in pellet-count surveys on 5 Key islands in 2009 to estimate occupancy and detection probability are indicated in dark gray.

zone was found between the coastal margins and interior uplands that included brackish saltmarsh at lower elevations transitioning to buttonwood (Conocarpus erectus) cover nearer to upland areas (Faulhaber et al. 2007, Schmidt 2009). Dominant herbaceous species in the low marsh transition zone included shoregrass (Monanthocloe littoralis), sea purslane (Sesuvium maritimum), American glasswort (Salicornia virginica), and saltwort (Batis maritime). Sea daisies (Borrichia spp.) and seashore dropseed (Sporobolus virginicus) were dominant in the mid-marsh transition, while gulf chord grass (Spartina spartinae), and fringe-rush (Fimbristylis spp.) were found in the high marsh transition zone (Forys 1995). Pine rockland (slash pine, Pinus elliottii), hardwood hammock, and freshwater marshes dominated by sawgrass (Cladium jamaicense) comprised upland vegetation communities.

We focused data collection on those habitat types recognized as suitable for marsh rabbits, based on the work of Howe (1988), Forys (1995), Forys et al. (1996), United States Fish and Wildlife Service (1999), Faulhaber et al. (2007), and others. Generally, habitat deemed suitable for marsh rabbits included saltmarsh transition zones and upland freshwater marshes with herbaceous ground cover. Within areas of suitable habitat, study patches were delineated by major roads, permanent water bodies, or the intersection of other vegetation types considered unsuitable for sustaining rabbit populations (Faulhaber et al. 2007).

\section{METHODS}

We used fecal pellet detection survey methods designed to estimate site occupancy of marsh rabbits. Revised survey methods were implemented in 2009 as the basis for longterm monitoring under an adaptive management program in the Florida Keys. To estimate detection probability (the probability of detecting presence of marsh rabbits in occupied habitat patches), we conducted replicate pellet surveys in multiple plots within a patch (i.e., sampling was replicated over space rather than time). From February to June, 2009, we randomly located, with replacement (Kendall and White 2009), 127 circular plots (12 m diameter) in 36 rabbit habitat patch sites on the 5 keys included in our study. The choice of a $12-\mathrm{m}$ diameter plot reflected a compromise between 1) an area small enough to yield high detection probabilities (given the presence of pellets in the plot) for better precision of occupancy estimates and 2) a large enough area as to have a non-negligible probability of containing pellets (given presence of rabbits in the patch). We based patch location and delineation on historical (Howe 1988, Forys 1995) and more recent (Faulhaber et al. 2007) marsh rabbit patch surveys. We used both habitat representativeness and logistical considerations with regard to sample size as criteria to select the islands and patches for inclusion in our study. Patch size was highly variable (0.12-51.2 ha) with a mean area of 7.6 ha. We attempted to standardize survey effort by maintaining a constant proportion of the number of plots relative to patch area. Because $\geq 2$ plots were required to estimate detection probability, small patches had higher plot densities (larger numbers of plots per unit patch area). Larger patches had smaller plot densities due to logistical limitations on the number of plots we could survey. Unequal survey effort among patches can be accommodated in occupancy models. 
Plots were systematically searched by one trained observer to detect marsh rabbit fecal pellets. No time constraint was imposed and plots with dense ground cover were searched as long as $40 \mathrm{~min}$. Because we employed spatial replicates to obtain detection histories within patches, we surveyed each plot only once unless plot selection with replacement resulted in overlapping sample areas. In such cases, a second visit followed completion of surveying the remaining plots in the habitat patch.

For data analysis, the unit of inference for marsh rabbit occupancy and detection was the individual habitat patch. We estimated site occupancy $(\psi)$ and detection probability $(p)$ over one season for the marsh rabbit following the methods of MacKenzie et al. (2003, 2006). Two occupancy states are possible for each site: occupied (corresponding probability is $\psi)$ and unoccupied $(1-\psi)$. Conditional on a site being occupied, the probabilities that the species is detected and not detected on a particular sample plot within that site are given by $p$ and $1-p$, respectively. We considered multiple survey plots within each patch as independent spatial replicates and as the basis for estimating $p$. Estimating detection probability using spatial sub-units may sometimes violate closure assumptions, due to nonavailability of rabbits in all survey plots (Kendall and White 2009). Detection probability, in this case, is the product of the probability that $\geq 1$ individuals of a species were available in a plot, given that the patch was occupied, and the probability that the species was detected, given that individuals were available for detection in the plot. Selection of sub-unit plots without replacement may lead to biased estimates of $p$ and $\psi$ (Kendall and White 2009). By selecting plots randomly and with replacement in each habitat patch, we reduced the possibility of inducing bias in $p$ and in $\psi$. We continue to refer to $p$ as detection probability, recognizing that it represents effective detection and that its magnitude is reduced by the probability that rabbits are not always available in a given plot, given that they are present in the patch.

We evaluated the influence of site characteristics on probabilities of occupancy and detection by modeling these factors as parameter covariates using a logit link function. Due to the limited amount of occupancy data that we could collect in one season, we limited our analysis to a priori hypotheses of 2 potential explanatory variables influencing occupancy and detection probability: 1) patch location with respect to coastal or inland vegetation communities and 2) patch area. We recorded patch location as binary, with a value of 1 (coastal) for patches characterized by presence of halophytic vegetation typical of saltmarsh transition zones and a value of 0 (inland) for patches characterized by upland (freshwater) marshes and their forest ecotones.

Patch size was the second site characteristic hypothesized to influence marsh rabbit occupancy (Faulhaber et al. 2007, Schmidt 2009, but see Forys and Humphrey 1999). Although a shape index ratio (patch perimeter to area) has previously been used to investigate the effects of patch edge on local persistence of the marsh rabbit (Schmidt 2009), we used total patch area due to ease of interpreting incremental effects of patch size on probability of occupancy. To avoid possible issues with model convergence caused by the large variation in habitat patch area, we standardized size by subtracting individual patch area from the mean patch-size and dividing by the standard deviation (standard normal transformation) across all patches included in the analysis. Sampling intensity (number of sample plots per unit patch area) was lower for large patches, prompting us to also investigate the possibility that detection probability was a decreasing function of patch size. This relationship between patch size and detection probability may arise as a function of rabbit density and, thus, availability to be detected in a given sub-patch plot. If larger patches support rabbits in lower densities, the average availability per plot may decrease regardless of plot number, thereby effectively decreasing estimates of detection probability.

The global model included both covariates (patch size and location) in estimating site occupancy $(\psi)$ and detection probability $(p)$ but did not include interaction terms. We modeled covariate data with a linear-logistic model:

$$
\operatorname{logit}\left(\theta_{i}\right)=\beta_{0}+\beta_{1} \times\left(\frac{\mathrm{Ha}_{i}-\overline{\mathrm{Ha}}}{\mathrm{SD}(\mathrm{Ha})}\right)+\beta_{2} \times \mathrm{Loc}_{i}
$$

where $\theta_{i}$ represents the parameter of interest for patch $i$, either occupancy $\left(\psi_{i}\right)$ or detection probability $\left(p_{i}\right)$. The covariate $\mathrm{Ha}_{i}$ is the area (ha) of individual patch $i$ and Loc $_{i}$ is a binary site location variable for coastal $=1$ and inland $=0$ patches. The intercept parameter $\beta_{0}$ represents the baseline value of occupancy or detection probability for inland patches of average size.

We applied single-season models to the rabbit detection history data using Program PRESENCE (Hines 2008). We tested all possible model combinations $(n=16)$ of habitat variables on $\psi$ and $p$ in a balanced design where each covariate was distributed equally among the candidate model set (Table 1). We did not include in the candidate set a model that allowed $p$ to vary by survey because sampling was spatially replicated and temporally unrelated across sites (MacKenzie et al. 2006). We assessed possible overdispersion of parameters for the global model using a parametric bootstrap goodness-of-fit test with 10,000 bootstrap iterations (MacKenzie and Bailey 2004). After correcting for lack of fit through computing an overdispersion parameter, $\hat{c}$, we used a quasi-likelihood-based information theoretic approach (Burnham and Anderson 2002) for model selection, computing the quasi-likelihood Akaike Information Criterion for small sample sizes $\left(\mathrm{QAIC}_{c}\right)$. Because there is not yet consensus among practitioners in determining effective sample sizes for occupancy models, we calculated $\mathrm{QAIC}_{c}$ using the number of patches $(n=36)$ as a conservative estimate for effective sample size (MacKenzie et al. 2006). We ranked models by their $\mathrm{QAIC}_{c}$ values and considered those with the lowest values more parsimonious. We used $\mathrm{QAIC}_{c}$ weights to compute model-averaged estimates and variances of real parameter values for occupancy and detection probability conditional on the results of all 16 likelihood models. Model-averaged variance is a function of both the variance of the parameter associated with each 
Table 1. The set of a priori models fit to pellet count data for Lower Keys marsh rabbit surveyed in 5 Florida Keys, USA, in 2009. We estimated occupancy ( $\psi$ ) and detection $(p)$ probabilities as functions of patch size (in hectares, $\mathrm{Ha}$ ) and patch location (coastal or inland, Loc). Additive covariate relationships are indicated by + (i.e., $\mathrm{Ha}+$ Loc). We based model selection on Akaike's Information Criterion (AIC) and corrected for overdispersion $(\hat{c}=1.51)$ and small sample size, yielding quasi-AIC $\left(\mathrm{QAIC}_{c}\right)$ values. We used the difference in support relative to the top-ranked model $\left(\Delta \mathrm{QAIC}_{c}\right), \operatorname{AIC}$ model weight $\left(w_{i}\right)$, relative model likelihood (Lk.hd.), the number of model parameters $(K)$, and twice the negative log-likelihood value $(-2 l)$ to compare among competing models.

\begin{tabular}{|c|c|c|c|c|c|c|}
\hline Model & $\mathrm{QAIC}_{c}$ & $\Delta$ QAIC $_{c}$ & $w_{i}$ & Lk.hd. & $K$ & $-2 l$ \\
\hline$\psi(\mathrm{Ha}+\mathrm{Loc}), p()$. & 99.83 & 0 & 0.261 & 1 & 4 & 136.54 \\
\hline$\psi(\mathrm{Loc}), p()$. & 100.32 & 0.49 & 0.205 & 0.783 & 3 & 141.10 \\
\hline$\psi(\mathrm{Ha}+\mathrm{Loc}), p(\mathrm{Loc})$ & 102.05 & 2.22 & 0.086 & 0.330 & 5 & 135.80 \\
\hline$\psi(\mathrm{Loc}), p(\mathrm{Loc})$ & 102.07 & 2.24 & 0.085 & 0.326 & 4 & 139.91 \\
\hline$\psi(\mathrm{Ha}+\mathrm{Loc}), p(\mathrm{Ha})$ & 102.28 & 2.45 & 0.077 & 0.294 & 5 & 136.14 \\
\hline$\psi(),. p(\mathrm{Loc})$ & 102.33 & 2.5 & 0.075 & 0.287 & 3 & 144.13 \\
\hline$\psi(\mathrm{Loc}), p(\mathrm{Ha})$ & 102.68 & 2.85 & 0.063 & 0.241 & 4 & 140.83 \\
\hline$\psi(),. p(\mathrm{Ha}+\mathrm{Loc})$ & 104.30 & 4.47 & 0.028 & 0.107 & 4 & 143.28 \\
\hline$\psi(\mathrm{Ha}), p(\mathrm{Loc})$ & 104.54 & 4.71 & 0.025 & 0.095 & 4 & 143.64 \\
\hline$\psi(\mathrm{Loc}), p(\mathrm{Ha}+\mathrm{Loc})$ & 104.76 & 4.93 & 0.022 & 0.085 & 5 & 139.88 \\
\hline$\psi(\mathrm{Ha}+\mathrm{Loc}), p(\mathrm{Ha}+\mathrm{Loc})$ & 104.82 & 4.99 & 0.022 & 0.083 & 6 & 135.61 \\
\hline$\psi(),. p()$. & 105.27 & 5.44 & 0.017 & 0.066 & 2 & 152.17 \\
\hline$\psi(\mathrm{Ha}), p()$. & 105.28 & 5.45 & 0.017 & 0.066 & 3 & 148.58 \\
\hline$\psi(\mathrm{Ha}), p(\mathrm{Ha}+\mathrm{Loc})$ & 106.95 & 7.12 & 0.007 & 0.028 & 5 & 143.18 \\
\hline$\psi(),. p(\mathrm{Ha})$ & 107.65 & 7.82 & 0.005 & 0.020 & 3 & 152.16 \\
\hline$\psi(\mathrm{Ha}), p(\mathrm{Ha})$ & 107.73 & 7.9 & 0.005 & 0.019 & 4 & 148.44 \\
\hline
\end{tabular}

individual model and the discrepancy between modelspecific and the weighted mean parameter estimates (Burnham and Anderson 2002).

\section{RESULTS}

We placed $2-10$ plots $(\bar{x}=3.65, \mathrm{SD}=5.8$ plots $/ \mathrm{ha})$ in each of the 36 patches surveyed. Naïve estimates of occupancy were 0.86 for inland patches $(95 \% \mathrm{CI}=0.67,1.0)$ and 0.32 for coastal patches $(\mathrm{CI}=0.12,0.52)$, with an aggregated estimate of 0.53 of patches occupied $(\mathrm{CI}=0.36,0.69)$. A goodness-of-fit test suggested some data overdispersion, and we applied a $\hat{c}$-value of 1.51 for model selection and variance inflation. The most parsimonious model, with a $\mathrm{QAIC}_{c}$ weight (w) of 0.26 , was $\psi(\mathrm{Ha}+\operatorname{Loc}) p($.$) , indicating that$ both location and patch size influenced occupancy but that detection probability per plot did not differ between locations (Table 1). Under this model, the probability of detecting rabbit sign on a sample plot, given rabbits were present in the patch, was $0.46(95 \% \mathrm{CI}=0.35,0.57)$. The average rate of detection suggests that the compromise we made in selecting circular plots of $12-\mathrm{m}$ diameter was appropriate for our study and this plot size will serve for future monitoring activities. Location was included as an explanatory variable for occupancy in the top 5 models, and the summed weight for all models including location (with respect to occupancy) was 0.82 . Patch size was included in only 3 of the top 5 ranking models, with parameter coefficients for patch size all estimated to be positive and with a combined weight of 0.50 across all models. The third-ranked model $(w=0.09)$ included probability of detection as a function of location; the total evidence weight for location as a factor influencing detection probability was 0.35 (Table 1).

Under the top-ranked model $[(\psi(\mathrm{Ha}+\mathrm{Loc}) p()$.$] , prob-$ ability of occupancy for coastal sites of mean patch size was 0.48 , with an approximate $95 \%$ confidence interval (based on a normal distribution) of $0.18-0.79$ (estimates of model coefficients for the top-ranked model are provided in Table 2). As expected, we estimated parameter $\beta_{1}$ to be positive, reflecting an increase in the probability of occupancy with larger patch sizes (Table 2). Predicted occupancy in coastal areas was approximately 0.21 for the smallest patches $(0.12 \mathrm{ha})$ but increased steeply to nearly 1.0 for patches $>30$ ha (Fig. 2). Because we detected rabbit sign at nearly all inland sites (12 of 14 locations), we estimated occupancy for inland sites to be 1.0 regardless of patch size (Table 2, Fig. 2). Model-averaged estimates of real occupancy probabilities across the set of 16 models was $0.98(\mathrm{SE}=0.092)$ for plots located in inland patches and $0.53(\mathrm{SE}=0.178)$ for coastal plots.

We evaluated possible differences in detection probabilities between coastal and inland sites based on the third-ranked model, $\psi(\mathrm{Ha}+\mathrm{Loc}), p(\mathrm{Loc})($ Table 3$)$. Estimates of detec-

Table 2. Linear-logistic coefficient estimates for occupancy probability of Lower Keys Marsh rabbits surveyed in the Florida Keys, USA, in 2009. Parameter estimates are from the best supported model, $\psi(\mathrm{Ha}+\mathrm{Loc}), p($.$) , in which the probability of occupancy (\psi)$ was modeled as a function of patch size $(\mathrm{Ha})$ and location (coastal vs. inland, Loc), and detection probability $(p)$ was independent of patch size or location;

$\operatorname{logit}\left(\psi_{i}\right)=\beta_{0}+\beta_{1} \times\left(\left(\mathrm{Ha}_{i}-\overline{\mathrm{Ha}}\right) /(\mathrm{SD}(\mathrm{Ha}))\right)+\beta_{2} \times \mathrm{Loc}_{i}$, where $\mathrm{Loc}_{i}=1$ (coastal) and $\mathrm{Loc}_{i}=0$ (inland). We based asymptotic upper and lower 95\% confidence bounds ( $2.50 \%$ and $97.5 \%$, respectively) of the estimates on a normal distribution.

\begin{tabular}{lrrrr}
\hline Logit $\left(\beta_{i}\right)$ & Estimate & SE & $\mathbf{2 . 5 0 \%}$ & 97.50\% \\
\hline$\beta_{0}$ & 26.844 & 3.919 & 19.162 & 34.527 \\
$\beta_{1}$ & 1.987 & 1.410 & -0.772 & 4.747 \\
$\beta_{2}$ & -26.925 & 3.919 & -34.607 & -19.243 \\
\hline
\end{tabular}




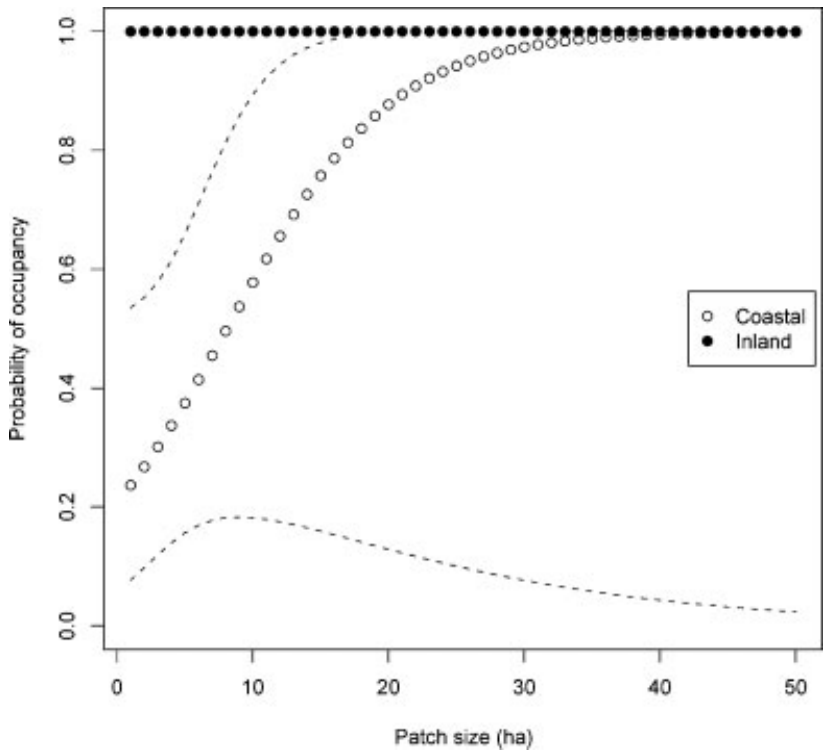

Figure 2. Occupancy probabilities for Lower Keys marsh rabbit, estimated from pellet-count surveys in the lower Florida Keys, USA, in 2009. Occupancy estimates are adjusted for imperfect detection probability at coastal and inland patches, conditional on patch size. Dashed lines indicate $95 \%$ confidence intervals for coastal sites.

tion probability were higher for plots located in inland patches $(0.49$, CI: $0.36-0.62)$ than for those in coastal patches (0.38, CI: $0.21-0.59)$, but there was substantial overlap of $95 \%$ confidence intervals. Model-averaged real estimates for detectability across all models was 0.47 $(\mathrm{SE}=0.077)$ for inland plots and $0.40(\mathrm{SE}=0.092)$ for plots located in coastal patches.

\section{DISCUSSION}

We found strong evidence that naïve estimates of patch occupancy for the Lower Keys marsh rabbit were negatively biased compared to estimates produced under a likelihoodbased occupancy model in which the probability of detection was quantified by surveying replicate plots in each habitat patch. Because we estimated detection probability to be $<0.5$ under both model-averaged results and the most parsimo-

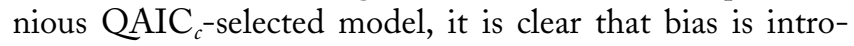
duced by ignoring imperfect detection is non-trivial; accounting for detectability can, therefore, greatly improve occupancy estimates. Testing for differences in occupancy

Table 3. Linear-logistic parameter estimates for detection probability of Lower Keys Marsh rabbits in the Florida Keys, USA, in 2009, under the third-ranked model, $\psi(\mathrm{Ha}+\mathrm{Loc}), p(\mathrm{Loc})$, in which the probability of occupancy $(\psi)$ was a function of patch size $(\mathrm{Ha})$ and location (coastal vs. inland, Loc) and detection probability $(p)$ was a function of location; $\operatorname{logit}\left(p_{i}\right)=\alpha_{0}+\alpha_{1}\left(\mathrm{Loc}_{i}\right)$, where $\operatorname{Loc}_{i}=1$ (coastal) and $\operatorname{Loc}_{i}=0$ (inland). We based asymptotic upper and lower $95 \%$ confidence bounds $(2.50 \%$ and $97.5 \%$, respectively) of the estimates on a normal distribution.

\begin{tabular}{lcccc}
\hline Logit $\left(\alpha_{\boldsymbol{i}}\right)$ & Estimate & SE & $\mathbf{2 . 5 0 \%}$ & $\mathbf{9 7 . 5 0 \%}$ \\
\hline$\alpha_{0}$ & -0.036 & 0.270 & -0.565 & 0.492 \\
$\alpha_{1}$ & -0.434 & 0.509 & -1.432 & 0.564 \\
\hline
\end{tabular}

and detectability between major vegetation classifications (coastal vs. inland) provided evidence that detection probability was equivalent between habitat locations and patches of different sizes. Assuming equal detection probability among patches, we concluded that location was a strong determinant of marsh rabbit occupancy rate. Upland vegetation patches of average size were approximately twice as likely to contain rabbits as coastal patches. Probability of occupancy in coastal patches was positively correlated with patch size, with an apparent ecological threshold (approx. $30 \mathrm{ha}$ ) where probability of occupancy increased to 1.0.

Our naïve estimates of patch occupancy were similar to those reported from previous surveys: 0.71 in transition zone habitat (Forys and Humphrey 1999) and 0.50 and 0.51 in surveys from 1988 to 1995 and from 2001 to 2005, respectively (Faulhaber et al. 2007). Given the observed similarities, it is reasonable to assume that estimates produced by earlier studies have suffered from bias due to the failure to consider effects of imperfect detection. Faulhaber et al. (2007) reported occupancy by marsh rabbits in numerous habitat patches previously considered as unoccupied or of unsuitable habitat, as well as the apparent extirpation of marsh rabbits from 2 small islands. Both Faulhaber et al. (2007) and Forys and Humphrey (1999) surveyed patches on multiple occasions within years but attributed the sequence of pellet detection and non-detection on an individual patch to the site being inconsistently occupied rather than to an imperfect observation process. It is possible that by considering issues of detectability in a statistical framework, the studies of Faulhaber et al. (2007) and Forys and Humphrey (1999) could have evaluated the probability of occupancy for 108 patches classified as unoccupied and, importantly, the probability of incorrectly declaring patches or islands to have been extirpated of marsh rabbits.

Our intention in developing a single-season occupancy model, using a revised sampling design to estimate detection probability, was to apply the inference gained to build more informative patch dynamics models for the Lower Keys marsh rabbit. These models will be used to anticipate the response of rabbits to habitat management and to guide annual management decisions through implementation of a formal adaptive management program (Williams et al. 2002). Accordingly, we will use our estimates of detection probability to revisit historical survey records and draw inference on marsh rabbit occupancy dynamics over time. Because historical data were not collected to address issues of non-detection, we are left to speculate that detection probability estimated from 2009 is applicable to data collected previously. The consistency of search methods across studies and the similarity of naive occupancy estimates between earlier surveys and our study provide some confidence in the assumption that detection probability has remained stable. Although we have no way to test if such an assumption is true, our only choice is to use the best available information when building models to predict marsh rabbit response to each potential management action to select the best long-term strategy for increasing species persistence. Maintaining the revised pellet sampling protocol will even- 
tually allow investigators to test the assumption of constant detection probability and, therefore, increase the strength of inference regarding the process of local extinction and colonization in marsh rabbit patches.

As an endangered lagomorph, the Lower Keys marsh rabbit is by no means unique. Nearly $25 \%$ of all rabbits, hares, and pikas are threatened with extinction, and populations of many additional species are estimated to be declining (see Smith 2008). Status surveys and monitoring of these species and populations are critical for their conservation and management. Our study design and analytical approach allow for quantification of detection probability when estimating distribution or occupancy and may be appropriate for many lagomorphs and other cryptic species inhabiting diverse or dynamic habitats.

\section{MANAGEMENT IMPLICATIONS}

Predicting rabbit responses to management decisions will likely take the form of dynamic occupancy models, where transitions of patches from one state to another (e.g., colonization of unoccupied sites, extinction of occupied sites) are modeled as direct functions of habitat characteristics and as indirect functions of the influence of management actions on habitat state. Multi-season occupancy models must be developed to estimate colonization and extinction parameters. Although a long-term monitoring program for the marsh rabbit has been operating at the National Key Deer Refuge, the 2009 survey was the first designed with the objective of quantifying detection probability to eliminate bias in patch occupancy estimates. With an understanding of the effect of management actions on habitat dynamics, we will then have the means to construct models to guide a policy of optimal management decisions through an adaptive management framework.

\section{ACKNOWLEDGMENTS}

We thank L. Bailey, S. Converse, and A. O'Connell for constructive comments on earlier drafts of this manuscript and $\mathrm{J}$. Hines with assistance in producing model-averaged variance estimates. Use of trade, product, or firm names does not imply endorsement by the United States Government.

\section{LITERATURE CITED}

Burnham, K. P., and D. R. Anderson. 2002. Model selection and multimodel inference: a practical information-theoretic approach. Second edition. Springer-Verlag, New York, New York, USA.

Carrete, M., J. A. Sanchez-Zapata, J. E. Martinez, M. A. Sanchez, and J. F. Calvo. 2002. Factors influencing the decline of a Bonelli's eagle Hieraaetus fasciatus population in southeastern Spain: demography, habitat or competition? Biodiversity and Conservation 11:975-985.

Collins, C. D., R. D. Holt, and B. L. Foster. 2009. Patch size effects on plant species decline in an experimentally fragmented landscape. Ecology 90:2577-2588.

Conroy, M. J., R. J. Barker, P. W. Dillingham, D. Fletcher, A. M. Gormley, and I. M. Westbrooke. 2008. Application of decision theory to conservation management: recovery of Hector's dolphin. Wildlife Research 35:93102.

Diamond, J. M. 1975. Assembly of species communities. Pages 242-444 in M. L. Cody, and J. M. Diamond, editors. Ecology and evolution of communities. Harvard University Press, Cambridge, Massachusetts, USA.
Doherty, P. F., T. Boulinier, and J. D. Nichols. 2003. Local extinction and turnover rates at the edge and interior of species ranges. Annales Zoologici Fennici 40:145-153.

Faulhaber, C. A., N. D. Perry, N. J. Silvy, R. R. Lopez, P. A. Frank, P. T. Hughes, and M. J. Peterson. 2007. Updated distribution of the Lower Keys marsh rabbit. Journal of Wildlife Management 71:208-212.

Forys, E. A. 1995. Metapopulations of marsh rabbits: a population viability analysis for the Lower Keys marsh rabbit (Sylvilagus palustris hefneri). Dissertation, University of Florida, Gainesville, USA.

Forys, E. A., and S. R. Humphrey. 1997. Comparison of 2 methods to estimate density of an endangered lagomorph. Journal of Wildlife Management 61:86-92.

Forys, E., and S. R. Humphrey. 1999. The importance of patch attributes and context to the management and recovery of an endangered lagomorph. Landscape Ecology 14:177-185.

Forys, E. A., P. A. Frank, and R. S. Kautz. 1996. Recovery actions for the Lower Keys marsh rabbit, silver rice rat, and Stock Island tree snail. Florida Game and Fresh Water Fish Commission, Office of Environmental Services, Tallahassee, USA.

Gotelli, N. J. 2000. Null model analysis of species co-occurrence patterns. Ecology 81:2606-2621.

Hanski, I. 1991. Single-species metapopulation dynamics - concepts, models and observations. Biological Journal of the Linnean Society 42:17-38.

Hanski, I. 1992. Inferences from ecological incidence functions. American Naturalist 139:657-662.

Hines, J. E. 2008. PRESENCE2-Software to estimate patch occupancy and related parameters. USGS-PWRC. <http://www.mbr-pwrc.usgs. gov/software/presence.html>. Accessed 28 Apr 2010.

Howe, S. E. 1988. Lower Keys marsh rabbit status survey. United States Fish and Wildlife Service, Jacksonville Field Station, Jacksonville, Florida, USA.

Kendall, W. L., and G. C. White. 2009. A cautionary note on substituting spatial subunits for repeated temporal sampling in studies of site occupancy. Journal of Applied Ecology 46:1182-1188.

Komonen, A., T. Tikkamaki, N. Mattila, and J. S. Kotiaho. 2008. Patch size and connectivity influence the population turnover of the threatened chequered blue butterfly, Scolitantides orion (Lepidoptera: Lycaenidae). European Journal of Entomology 105:131-136.

Kroll, A. J., K. Risenhoover, T. McBride, E. Beach, B. J. Kernohan, J. Light, and J. Bach. 2008. Factors influencing stream occupancy and detection probability parameters of stream-associated amphibians in commercial forests of Oregon and Washington, USA. Forest Ecology and Management 255:3726-3735.

Mace, G. M., and R. Lande. 1991. Assessing extinction threats—toward a reevaluation of IUCN threatened species categories. Conservation Biology 5:148-157.

MacKenzie, D. I., and L. L. Bailey. 2004. Assessing the fit of site-occupancy models. Journal of Agricultural Biological and Environmental Statistics 9:300-318.

MacKenzie, D. I., and W. L. Kendall. 2002. How should detection probability be incorporated into estimates of relative abundance? Ecology 83:2387-2393.

MacKenzie, D. I., and J. D. Nichols. 2004. Occupancy as a surrogate for abundance estimation. Animal Biodiversity and Conservation 27:461467.

MacKenzie, D. I., J. D. Nichols, J. E. Hines, M. G. Knutson, and A. B. Franklin. 2003. Estimating site occupancy, colonization, and local extinction when a species is detected imperfectly. Ecology 84: 2200-2207.

MacKenzie, D. I., L. L. Bailey, and J. D. Nichols. 2004. Investigating species co-occurrence patterns when species are detected imperfectly. Journal of Animal Ecology 73:546-555.

MacKenzie, D. I., J. D. Nichols, J. A. Royle, K. H. Pollock, J. E. Hines, and L. L. Bailey. 2006. Occupancy estimation and modeling: inferring patterns and dynamics of species occurrence. Elsevier, San Diego, California, USA.

Martin, J., C. L. McIntyre, J. E. Hines, J. D. Nichols, J. A. Schmutz, and M. C. MacCluskie. 2009. Dynamic multistate site occupancy models to evaluate hypotheses relevant to conservation of Golden Eagles in Denali National Park, Alaska. Biological Conservation 142:2726-2731.

Martin, J., S. Chamaille-Jammes, J. D. Nichols, H. Fritz, J. E. Hines, C. J. Fonnesbeck, D. I. MacKenzie, and L. L. Bailey. 2010. Simultaneous modeling of habitat suitability, occupancy and relative abundance: case 
study of African Elephants in Zimbabwe. Ecological Applications 20:1173-1182.

Ovaskainen, O. 2002. Long-term persistence of species and the SLOSS problem. Journal of Theoretical Biology 218:419-433.

Pielke, R. A., and R. T. Conant. 2003. Best practices in prediction for decision-making: lessons from the atmospheric and earth sciences. Ecology 84:1351-1358.

Ross, M. S., J. J. O’Brien, and L. J. Flynn. 1992. Ecological site classification of Florida Keys terrestrial habitats. Biotropica 24:488-502.

Royle, J. A., J. D. Nichols, and M. Kéry. 2005. Modelling occurrence and abundance of species when detection is imperfect. Oikos 110:353-359.

Schmidt, P. M. 2009. Metapopulation ecology and recovery of the Lower Keys marsh rabbit. Dissertation, Texas A\&M University, College Station, USA.

Smith, A,T. 2008. Conservation of endangered lagomorphs. Pages 297-315 in P.C. Alves, N. Ferrand, and K. Hackländer, editors. Lagomorph biology: evolution, ecology and conservation. Springer-Verlag, Berlin, Germany.
Taylor B, 1991. Investigating species incidence over habitat fragments of different areas-a look at error estimation. Biological Journal of the Linnean Society 42:177-191.

United States Fish and Wildlife Service. 1990. Endangered and threatened wildlife and plants; endangered status for the Lower Keys marsh rabbit and threatened status for the Squirrel Chimney cave shrimp. Federal Register 55:25588-25591.

United States Fish and Wildlife Service. 2007. Lower Keys marsh rabbit (Sylvilagus palustris hefneri) 5-year review: summary and evaluation. U.S. Fish and Wildlife Service, Vero Beach, Florida, USA.

United States Fish and Wildlife Service. 1999. South Florida multi-species recovery plan. U.S. Fish and Wildlife Service, Atlanta, Georgia, USA.

Williams, B. K., J. D. Nichols, and M. J. Conroy. 2002. Analysis and management of animal populations: modeling, estimation, and decision making. Academic Press, San Diego, California, USA.

Associate Editor: Leonard Brennan. 